\title{
No Obesity Paradox-BMI Incapable of Adequately Capturing the Relation of Obesity with All-Cause Mortality: An Inception Diabetes Cohort Study
}

\author{
Mohammadreza Bozorgmanesh, ${ }^{1}$ Banafsheh Arshi, ${ }^{1}$ Farhad Sheikholeslami, ${ }^{1}$ \\ Fereidoun Azizi, ${ }^{2}$ and Farzad Hadaegh ${ }^{1}$ \\ ${ }^{1}$ Prevention of Metabolic Disorders Research Center, Research Institute for Endocrine Sciences (RIES), \\ Shahid Beheshti University of Medical Sciences, Tehran 1985717413, Iran \\ ${ }^{2}$ Endocrine Research Center, Research Institute for Endocrine Sciences (RIES), Shahid Beheshti University of Medical Sciences, \\ Tehran 1985717413, Iran
}

Correspondence should be addressed to Farzad Hadaegh; fzhadaegh@endocrine.ac.ir

Received 2 June 2014; Accepted 9 July 2014; Published 7 August 2014

Academic Editor: Mario Maggi

Copyright (C) 2014 Mohammadreza Bozorgmanesh et al. This is an open access article distributed under the Creative Commons Attribution License, which permits unrestricted use, distribution, and reproduction in any medium, provided the original work is properly cited.

\begin{abstract}
Background. To reconcile "the obesity paradox," we tested if (1) the contribution of anthropometric measures to mortality was nonlinear and (2) the confounding of hip circumference contributed to the obesity paradox recently observed among diabetic patients. Methods. We analyzed data of diabetic patients attending a community-based prospective, "Tehran lipid and glucose study." In the mortality analysis, anthropometric measures-body mass index (BMI), waist, and hip circumference-were assessed using Cox models incorporating cubic spline functions. Results. During 12990 person-years follow-up, BMI levels below 27 and those above $40 \mathrm{~kg} \cdot \mathrm{m}^{-2}$ were associated with increased mortality. When we added waist circumference to the BMI in the multivariate-adjusted model, the steepness of BMI-mortality association curve slope for values below $27 \mathrm{~kg} \cdot \mathrm{m}^{-2}$ increased, whereas the steepness of BMI-mortality association curve slope for values above this threshold decreased. Further adjusting the model for hip circumference, the steepness of the slopes of the association curve moved towards null on both extremes and no associations between BMI and all-cause mortality remained. Conclusion. BMI harbors intermixed positive and negative confounding effects on mortality of waist and hip circumference. Failing to control for the confounding effect of hip circumference may stymie unbiased hazard estimation and render conclusions paradoxical.
\end{abstract}

\section{Background}

The so-called "obesity paradox" has been observed in a variety of settings [1-5]. The U-shaped relationship of body mass index (BMI) with mortality has been demonstrated in general populations, yet, the definite excess mortality below $25 \mathrm{~kg} \cdot \mathrm{m}^{-2}$ has not been fully explained [6]. Researchers have defined a condition generally referred to as the metabolically obese normal weight phenotype. This phenotype is ascertained among normal weight individuals based on their BMI but present metabolic aspects of obesity. The condition has been observed to be increasingly prevailing over time $[3,7-9]$.
Along with the emergence of the concept of metabolically obese normal weight, the "obesity paradox" phenomenon came into notion $[10,11]$. Obesity was long known to contribute to mortality [2]. Examining nonlinear contribution of the anthropometric indices of obesity, however, unveiled a Ushaped association between obesity measures and mortality, particularly among patients with chronic disease like hypertension [12], kidney disease [13], and heart failure [14].

No other chronic disease is as known to be related to obesity as diabetes. The role of obesity in diabetes was so ingrained that some investigators suggested the term "DIABESITY (DIABEtes + obeSITY)" for both conditions [15]. However, what hinders the term to become extensively 
used are findings indicating that diabetic patients who lost weight during follow-up or were of normal weight at the baseline had a higher mortality than diabetic patients who were overweight or obese $[16,17]$. Participants of these studies had diabetes of unknown duration [18]. This makes it difficult to distinguish between the role of weight loss as an innocent bystander reflecting poorly controlled diabetes or as a mediator effectuating the impact of diabetes on death or as an independent risk factor [19]. To deal with this limitation, Carnethon et al. have conducted a study on a sample of participant with newly diagnosed diabetes to examine all-cause, cardiovascular, and noncardiovascular mortality. As such, the obesity status of the participants might not have been confounded by diabetes. They observed that among newly diagnosed diabetic patients, normal weight at the time of diagnosis resulted in twice the mortality rate overweight/obesity did. Arbitrarily defining normal weight/overweight/obese by discrediting BMI may lead to (1) an unrealistic step-function of risk that assumes homogeneity of risk within groups, (2) loss of power [20] inaccurate estimation, and (4) incomplete correction for confounding $[21,22]$. Furthermore, it has been shown that failing to account for the negative confounding (suppression) effect of hip circumference while studying obesity-related risk might lead to biased estimates [23].

Using an inception cohort of diabetic patients, we conducted the current study aiming to reconcile the "obesity paradox" recently observed among diabetic patients. This is an attempt to examine the apparently contradictory statements regarding the obesity-mortality association and to draw conclusions to either reconcile them or explain their existence. As such, we tested the hypotheses that (1) the contributions of anthropometric measures of obesity to mortality are nonlinear and (2) the confounding bias due to hip and waist circumference might have contributed to the "obesity paradox" among diabetic patients.

\section{Research Design and Methods}

2.1. Study Design. Detailed descriptions of the Tehran lipid and glucose study (TLGS) have been reported elsewhere [18]; in brief, the TLGS is a large scale, long term, communitybased prospective study performed on a representative sample of residents of district number 13 of Tehran, capital of Iran. Age and sex distributions of the population in the district were representative of the overall population of Tehran at the time of the baseline examination. Details of setting, measurements, and outcomes have been described in the supplementary file (see Supplementary File 1 available online at http://dx.doi.org/10.1155/2014/282089).

2.2. Statistical Methods. Findings on covariate variables are expressed as means (SD) and frequency (\%) for continuously and categorically distributed variables, respectively. We tested for trends across BMI tertiles by using the median in each tertile as a predictor. Statistical significance of trends across BMI tertiles was examined by implementing General Linear models. The flexible parametric model for survival analysis was used to plot mortality rates (hazard function) against followup time. We concentrated on continuous time-dependent effect using restricted cubic spline functions to model the baseline cumulative hazard. We fitted nested models with and without spline functions of the baseline hazard and estimated the deviance (D-statistics) for each model based on the maximum likelihood $\chi^{2}$. The statistical significance of the time-dependent effect was then determined by obtaining the difference in deviances of the two nested models (Gstatistic) based on the maximum likelihood $\chi^{2}$. Since we found no evidence of nonproportionality, the Log-Rank test and Cox test were used to examine the significance of trends in incident rates and hazard functions.

We set the statistical significance level at a two-tailed type I error of 0.05. All statistical analyses were performed using STATA version 12.0 (STATA, College Station, TX, USA).

We certify that all applicable institutional and governmental regulations concerning the ethical use of human volunteers were followed during this research. Informed written consent was obtained from all participants and the Ethical Committee of Research Institute for Endocrine Sciences approved this study. The investigations reported herein have been carried out in accordance with the principles of the Declaration of Helsinki as revised in 2000.

\section{Results}

3.1. Participants. Among participants aged $\geq 30$ years, we selected those not using glucose lowering agents but diagnosed to have new onset diabetes at the baseline examination and those who developed incident diabetes during any of the two consecutive follow-up examination cycles. Complete data on covariates were available for 1,322 of participants with the median follow-up time being 9.1 years.

3.2. Descriptive Data. Clinical and paraclinical characteristics of participants at the time when they were first diagnosed to have diabetes are stratified by tertiles of BMI in Table 1. Age of the participants at the time they were diagnosed to have diabetes ranged from 30 to 90 years.

No consistent trend in mortality rates was observed across tertiles of BMI. In fact the first $(P$ value $=0.056)$ and the third $(P$ value $=0.588)$ tertiles were observed to contribute to higher mortality rates than did the middle tertile (Table 2). As shown in Table 2, adjustment for waist circumference accentuated the HRs for all-cause mortality of the first but not third BMI tertiles as compared to the middle tertile. However, further adjustment for established CVD risk factors made the statistical significance disappear.

As shown in Figure 1, when we plotted the waist-tohip ratio (WHpR) against $\mathrm{BMI}$, the ratio was observed to increase with increasing levels of BMI up to the threshold of $\approx 27 \mathrm{~kg} \cdot \mathrm{m}^{-2}$, where the association started to reverse. That is, as BMI increased above $27 \mathrm{~kg} \cdot \mathrm{m}^{-2}$, the magnitude of increases in hip circumference levels was higher than those in waist circumference.

Figure 2 depicts the multivariate-adjusted nonlinear contribution of the BMI to all-cause mortality adjusted for CVD 
TABLE 1: Baseline characteristics of the participants with new-onset diabetes mellitus stratified by tertile of body mass index.

\begin{tabular}{|c|c|c|c|c|c|}
\hline & Tertile 1 & Tertile 2 & Tertile 3 & $P$ value ${ }^{*}$ & Total \\
\hline Number of participants & 441 & 441 & 440 & - & 1322 \\
\hline Median body mass index $\left(\mathrm{kg} \cdot \mathrm{m}^{-2}\right)$ & 24.9 & 28.9 & 33.8 & - & 28.9 \\
\hline Minimum body mass index $\left(\mathrm{kg} \cdot \mathrm{m}^{-2}\right)$ & 15.7 & 26.9 & 31.1 & - & 15.7 \\
\hline Maximum body mass index $\left(\mathrm{kg} \cdot \mathrm{m}^{-2}\right)$ & 26.9 & 31.1 & 57.7 & - & 57.7 \\
\hline \multicolumn{6}{|l|}{ Categorically distributed variables } \\
\hline Male & $259(0.59)$ & $213(0.48)$ & $118(0.27)$ & $<0.001$ & $590(0.45)$ \\
\hline Smoker & $67(0.15)$ & $55(0.13)$ & $32(0.07)$ & $<0.001$ & $154(0.12)$ \\
\hline Blood pressure lowering drug usage & $107(0.24)$ & $118(0.27)$ & $136(0.31)$ & $<0.001$ & $361(0.27)$ \\
\hline History of previous cardiovascular disease & $76(0.18)$ & $90(0.21)$ & $80(0.18)$ & 0.957 & $246(0.19)$ \\
\hline Assigned to life style modification intervention & $160(0.36)$ & $170(0.39)$ & $166(0.38)$ & 0.549 & $496(0.38)$ \\
\hline \multicolumn{6}{|l|}{ Continuously distributed variables } \\
\hline Age (years) & $55.22(11.96)$ & $53.48(11.69)$ & $52.25(10.93)$ & $<0.001$ & $53.65(11.59)$ \\
\hline Systolic blood pressure $(\mathrm{mmHg})$ & $127.40(20.77)$ & $130.02(22.75)$ & $132.52(21.79)$ & $<0.001$ & $129.97(21.86)$ \\
\hline Total cholesterol $\left(\mathrm{mmol} \cdot \mathrm{l}^{-1}\right)$ & $5.65(1.23)$ & $5.84(1.32)$ & $5.82(1.21)$ & 0.001 & $5.77(1.26)$ \\
\hline High-density lipoprotein cholesterol $\left(\mathrm{mmol} \cdot \mathrm{l}^{-1}\right)$ & $1.03(0.28)$ & $1.01(0.29)$ & $1.04(0.26)$ & 0.002 & $1.03(0.28)$ \\
\hline Waist circumference $(\mathrm{cm})$ & $87.87(8.05)$ & $97.31(6.98)$ & $106.03(8.44)$ & $<0.001$ & $97.06(10.87)$ \\
\hline Hip circumference $(\mathrm{cm})$ & $94.80(5.03)$ & $101.87(4.56)$ & $112.87(5.58)$ & $<0.001$ & $103.16(9.75)$ \\
\hline \multicolumn{6}{|l|}{ Outcome } \\
\hline All-cause mortality & $48(10.9)$ & $33(7.5)$ & $27(6.1)$ & 0.236 & $108(8.2)$ \\
\hline All-cause mortality rate, per (10 000 person-year) & $90.8(65.8-125.3)$ & $53.7(35.0-82.3)$ & $64.2(44.0-93.5)$ & 0.140 & $69.7(56.3-86.2)$ \\
\hline
\end{tabular}

Data are presented as mean (SD) or frequency (\%) for continuously and categorically distributed variables, respectively.

Mortality is presented as per 10000 person-years (95\% CIs).

* $P$ values were obtained from general linear models adjusted for age for independent variables; mortality rates were compared using Cox proportional hazard regression model.

TABLE 2: Contribution of different tertiles of the body mass index to all-cause mortality.

\begin{tabular}{|c|c|c|c|c|}
\hline & Hazard Ratio (95\% CIs) & Std. Err. & Wald $\chi^{2}$ & $P$ value \\
\hline \multicolumn{5}{|l|}{ Model 1} \\
\hline \multicolumn{5}{|l|}{ Body mass index $\left(\mathrm{kg} \cdot \mathrm{m}^{-2}\right)$} \\
\hline First tertile & $1.69(0.99-2.88)$ & 0.46 & 1.91 & 0.056 \\
\hline Second tertile & 1 [reference] & & & \\
\hline Third tertile & $1.17(0.66-2.07)$ & 0.34 & 0.54 & 0.588 \\
\hline \multicolumn{5}{|l|}{ Model 2} \\
\hline \multicolumn{5}{|l|}{ Body mass index $\left(\mathrm{kg} \cdot \mathrm{m}^{-2}\right)$} \\
\hline First tertile & $2.36(1.30-4.30)$ & 0.72 & 2.81 & 0.005 \\
\hline Second tertile & 1 [reference] & & & \\
\hline Third tertile & $0.96(0.52-1.77)$ & 0.30 & -0.12 & 0.904 \\
\hline Log-waist circumference $[\ln (\mathrm{cm})]$ & $20.35(1.62-254.79)$ & 26.24 & 2.34 & 0.019 \\
\hline \multicolumn{5}{|l|}{ Model 3} \\
\hline \multicolumn{5}{|l|}{ Body mass index $\left(\mathrm{kg} \cdot \mathrm{m}^{-2}\right)$} \\
\hline First tertile & $1.54(0.83-2.86)$ & 0.49 & 1.38 & 0.168 \\
\hline Second tertile & 1 [reference] & & & \\
\hline Third tertile & $1.49(0.80-2.76)$ & 0.47 & 1.25 & 0.211 \\
\hline Log-general cardiovascular risk & $2.81(2.11-3.75)$ & 0.41 & 7.02 & $<0.001$ \\
\hline Log-waist circumference $[\ln (\mathrm{cm})]$ & $0.65(0.04-10.50)$ & 0.93 & -0.30 & 0.763 \\
\hline
\end{tabular}

${ }^{\dagger}$ Cox proportional hazard models were used to calculate HRs and 95\% CIs. Model 1: adjusted with BMI. Model 2: adjusted with BMI and waist circumference. Model 3: adjusted with BMI, waist circumference, and general CVD risk. 


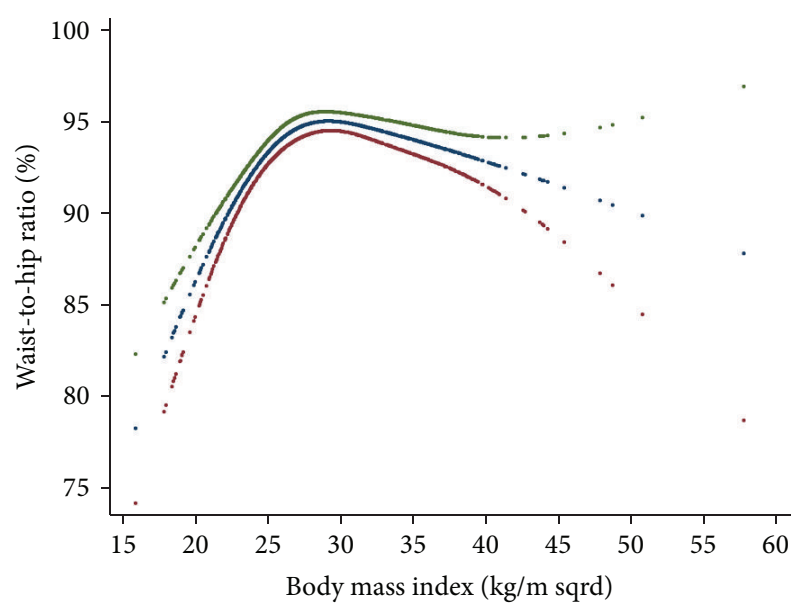

FIGURE 1: Inverse relationship of hip and waist circumference with body mass index.

risk factors only (a), CVD risk factors plus waist circumference (b), CVD risk factors plus hip circumference (c), and CVD risk factors plus both waist and hip circumference (d). As shown in the panel (a), BMI levels below 27 and above $40 \mathrm{~kg} \cdot \mathrm{m}^{-2}$ were associated with increased mortality. Our estimates, however, were not stable at the right-sided tail of the curve, possibly due to lack of statistical power.

As panel (b) depicts, when we added waist circumference to the BMI in the multivariate-adjusted model the steepness of BMI-mortality association curve slope for values below $27 \mathrm{~kg} \cdot \mathrm{m}^{-2}$ increased, whereas the steepness of BMImortality association curve slope for values above this threshold decreased. To examine if the increasing levels of BMI associated with decreasing trend in mortality up to BMI of $27 \mathrm{~kg} \cdot \mathrm{m}^{-2}$ were due to negative confounding effect of increasing hip circumference, we further adjusted the model for hip circumference and observed that the steepness of the slope of the association curve was observed to decrease considerably so that it was no longer statistically significant (panel (d)). As shown in panel (c), when we introduced hip circumference into the multivariable model already incorporating BMI, the steepness of BMI-mortality association curve slope for values below $27 \mathrm{~kg} \cdot \mathrm{m}^{-2}$ decreased, whereas, above this threshold, the steepness of the BMI-mortality association curve increased towards positive values.

Figure 3 depicts nonlinear contribution of waist (a) and hip (b) circumference to all-cause mortality. Waist and hip circumference were mutually adjusted for each other in addition to CVD risk factors and BMI. As waist circumference exceeded the threshold of $100 \mathrm{~cm}$, all-cause mortality increased in a linear fashion. As hip circumference increased, all-cause mortality decreased steadily in a linear fashion.

\section{Discussion}

4.1. Statement of Principal Findings. Using an inception cohort of adults with diabetes, we investigated the nonlinear contribution of the anthropometric indices of obesity to allcause mortality allowing for potential confounding bias due to established CVD risk factors. We observed that BMI, waist, and hip circumference were all associated with all-cause mortality in a curvilinear fashion.

The finding of interest was that even across values generally considered as normal, further decreases in the BMI was associated with increased mortality. Some observations regarding this finding were as follows.

(1) When the effect of waist circumference was taken into account, for values of BMI below $27 \mathrm{~kg} \cdot \mathrm{m}^{-2}$, the steepness of the slope increased. This implies that if waist circumference remains constant decreasing BMI would confer an excess mortality. Meanwhile, higher-than-normal values of BMI were not associated with increased mortality, except for extremely high values. That is, except for extremely high BMI values, increasing levels of BMI are safe as long as waist circumference remains constant.

(2) Conversely, when we controlled our analysis for hip circumference, the opposite was true. That is, when we introduced hip circumference into the multivariable model already incorporating BMI, the steepness of BMI-mortality association curve slope for values below $27 \mathrm{~kg} \cdot \mathrm{m}^{-2}$ decreased, whereas, above this threshold, the steepness of the BMI-mortality association curve increased. This implies that if hip circumference remains constant, decreasing levels of BMI will not result in excess mortality. In other words, BMI-associated mortality observed in the lower extreme is merely an artifact resulting from uncontrolled confounding bias due to hip circumference.

(3) An inverse U-shaped association was observed between the waist-to-hip ratio (WHpR) and BMI; that is, the ratio increased with increasing levels of BMI up to about $27 \mathrm{~kg} \cdot \mathrm{m}^{-2}$ and the association was reversed thereafter. In other words, the magnitude of increase in the waist circumference exceeded the increase in hip circumference up to BMI $\approx 27 \mathrm{~kg} \cdot \mathrm{m}^{-2}$; however, with higher BMI levels, the increase in BMI was accompanied by greater increase in hip circumference than in waist circumference. Changes in hip circumference parallels changes in waist circumference and BMI. However, in contrast to waist circumference and BMI, hip circumference inversely contributes to the mortality. As such, increased mortality due to increased waist circumference might have been counterbalanced by decreased mortality due to increases in hip circumference.

(4) To conclude, If we had not considered the effect of waist circumference, we would have overestimated the mortality associated with increasing values of BMI above normal values. Also, had we not taken the effect of hip into account, we would have overestimated the mortality associated with decreasing levels on BMI below normal values.

Aforementioned observations provided evidences to support the notion that it is not obesity that is paradoxically related to 


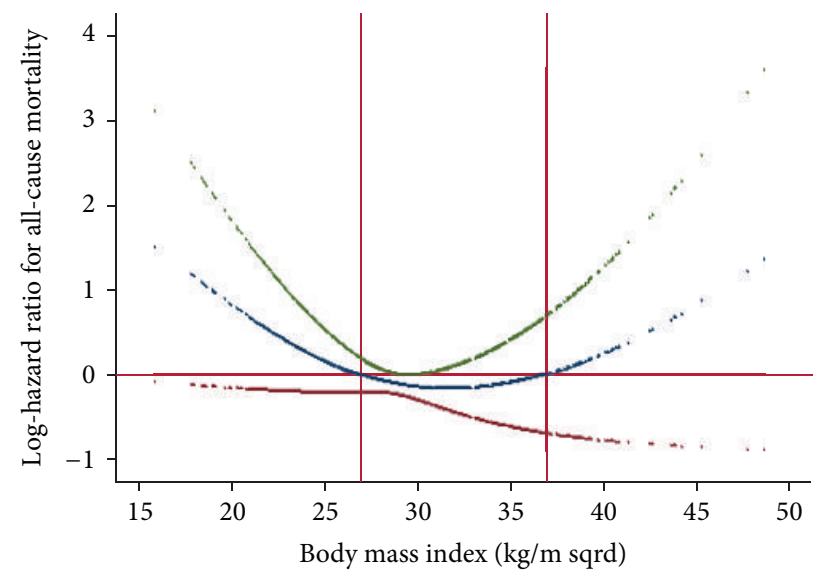

(a)

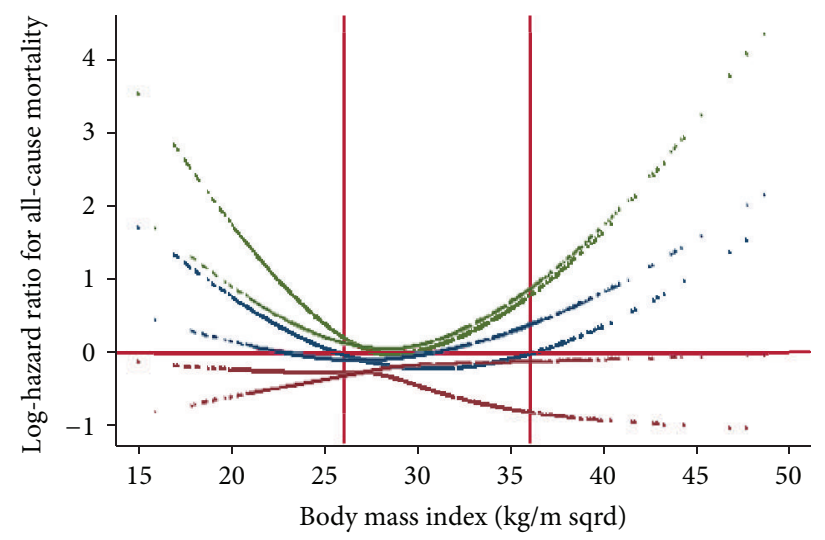

(c)

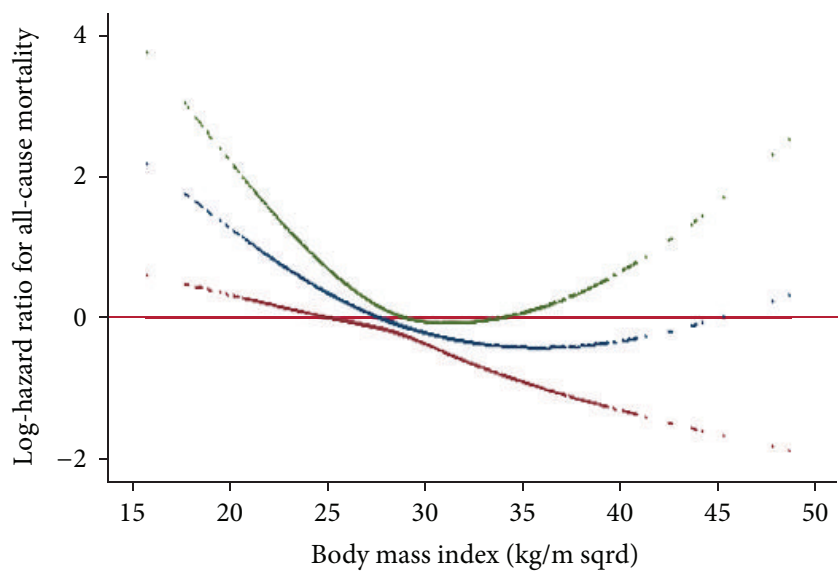

(b)

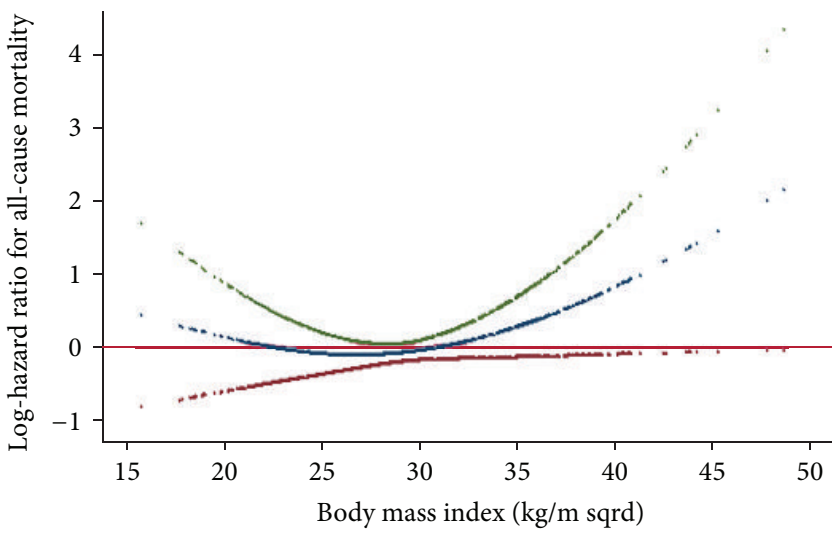

(d)

FIgURE 2: (a) Nonlinear contribution of body mass index to all-cause mortality. (b) Nonlinear contribution of body mass index to allcause mortality, allowing for waist circumference. (c) Nonlinear contribution of body mass index to all-cause mortality, allowing for hip circumference. (d) Nonlinear contribution of body mass index to all-cause mortality, allowing for both waist and hip circumference.

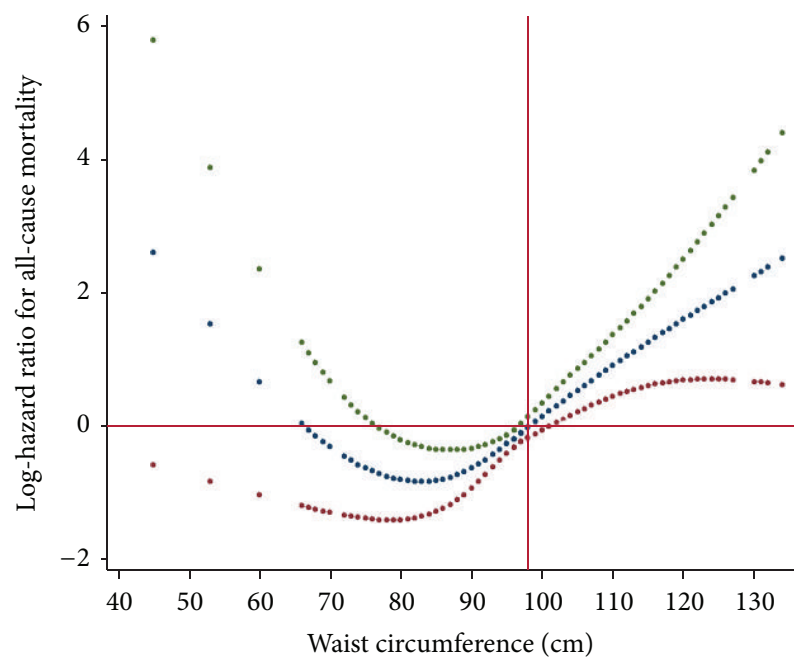

(a)

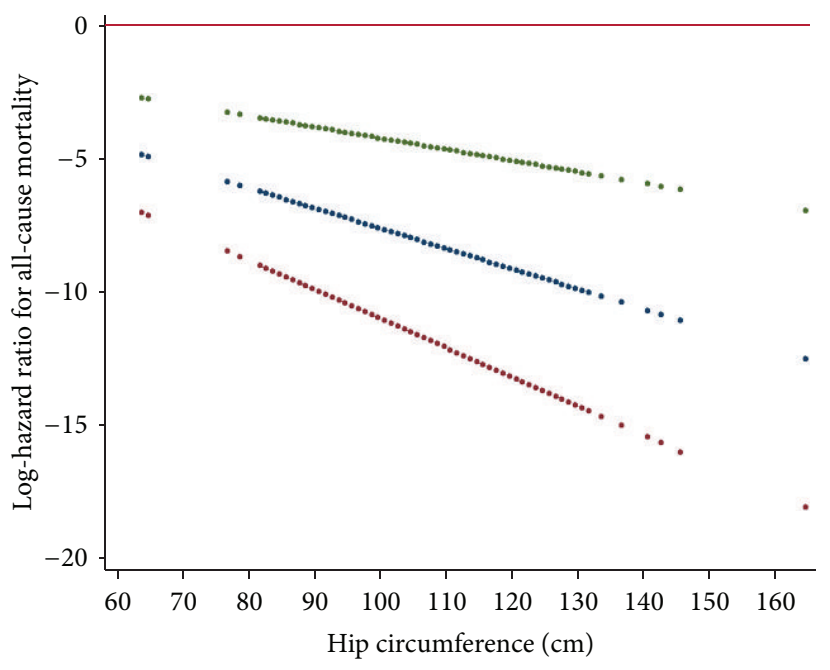

(b)

FIGURE 3: (a) Nonlinear contribution of waist circumference to all-cause mortality. (b) Linear contribution of hip circumference to all-cause mortality. 
all-cause mortality; rather it is BMI that harbors intermixed positive and negative confounding effects on mortality of waist and hip circumference, which have contradictory effect on all-cause mortality. To obtain an unbiased estimate of the general obesity-related health risk, in addition to waist circumference, it is crucial to control for the negative confounding effect of hip circumference. It is noteworthy that inasmuch as loss of kilograms inevitably includes loss of fatfree mass, BMI should not be ignored [24]. A better strategy, therefore, could be to use a measure of central adiposity like waist circumference and then control the association analyses for confounding bias originating from of BMI and hip circumference. Taking this approach, we observed that increases in central obesity above waist circumference of $100 \mathrm{~cm}$ were consistently associated with increase in all-cause mortality. Some investigators are interested in the fact that some obese individuals remain metabolically healthy and hypothesize that these individuals have a higher level of cardiovascular fitness than their metabolically abnormal obese peers and that such metabolically healthy obese individuals should demonstrate lower mortality rates [25]. In the current study, however, we adjusted our analyses for CVD risk factors so that the obesity-related mortality was independent of metabolic states. Although we have previously shown that obesity is not always associated with metabolic abnormality [26], herein, we observed that even if metabolic states remain constant increasing levels of central obesity not confounded by lean body mass or hip size were associated with increased mortality. What previous studies have referred to as the "obesity-paradox" could be reconciled in light of the fact that BMI is not a perfect measure of obesity. Although not necessarily in same direction, both body mass and fatness contribute to mortality. Such confounding bias originating from each part should be accounted for while studying obesity-related health risks, since these parameters exhibit covariation in many settings.

Some studies have suggested that waist circumference, either alone or in combination with BMI, may have a stronger relation to some health outcomes than BMI alone [27]. Waist circumference reflects abdominal or intraabdominal fat, and hip circumference reflects different aspects of body composition in the gluteofemoral region, that is, muscle, bone, and fat mass. The importance of waist and hip measurements, and the waist to hip ratio lies in the apparently different physical and metabolic characteristics of these two regions, and therefore the diverse clinical outcomes in individuals with a gynecoid (low waist to hip ratio, lower body obesity) or android (high waist to hip ratio, upper body obesity) body conformation. This may be due to the tendency for abdominal adipocytes to enlarge (hypertrophy) whereas subcutaneous femoral adipocytes increase in number (hyperplasia), perhaps due to increased levels of the adipogenic transcription factors; moreover hypertrophic adipocytes tend to be associated with dyslipidemia and insulin resistance [28].

4.2. Strength and Limitations. We used an inception cohort of diabetic patients which is now a standard for prognostic studies. Our patients were identified at an early and uniform point (inception) in the course of their disease. The cohort came from a large-population-based study of both sexes, with accurate and valid data on risk factors at baseline and continuous surveillance of mortality and CVD events based on standard criteria. In an attempt to reduce variability in the duration of new-onset diabetes, we restricted our analysis to participants who were not taking glucose lowering agents in the first examination. We used appropriate advanced statistical methods to capture nonlinear association of BMI, waist, and hip circumference with mortality. Both FPG and the standard 2h-PCPG levels were available in the TLGS for identifying incident diabetes cases. Controlling the analyses for established CVD risk factors made the estimates unlikely to be affected by the bias stemming from potential confounding effects of these risk factors. Some limitations of our study merit mentioning. First, the small number of incident events precluded stratification of analyses by sex. Second, large confidence intervals on the right-sided tail of the estimated curves imply lack of statistical power and our estimates might not have been stable at high BMI, hip, or waist circumference quantities. Third, despite our best efforts to control for bias from preexisting disease, it is likely that we did not eliminate such bias completely. Thus, the increased risk of death from specific causes associated with leanness may reflect preexisting, but unrecognized, disease processes. Fourth, due to our sample, we were not able to investigate association of measures of obesity with specific causes of death. Finally, the population studied was of Persian ancestry and, thus, cannot be readily extrapolated to other populations.

\subsection{Strengths and Weaknesses in relation to Other Studies.} Despite extensive use of BMI in research and clinical practice, there are very few studies testing its diagnostic accuracy and except for that of Romero-Corral et al. no study has done this in a large, multiethnic adult population representing men and women of many age strata [29]. Even though the association between obesity and mortality is unquestionable, multiple studies worldwide have shown that overweight individuals have similar or even lower mortality when compared to people classified as having normal body weight [30, 31]. Results of these studies have challenged the association of adiposity with mortality and cardiovascular disease, when they might just represent the intrinsic limitations of BMI to differentiate adipose tissue from lean mass in intermediate BMI ranges [29].

In a pooled analysis of five longitudinal cohort studies, Carnethon et al. observed that adults who were of normal weight at the time of diagnosing diabetes had higher mortality than their overweight/obese counterparts [17]. Using arbitrary definition for normal weighted, they were not able to discover the U-shaped associations. Furthermore, failing to account for suppressive effects of increasing levels of hip circumference, which parallels the increase in BMI, might have biased their estimated mortality hazard among overweight/obese patients towards the null and thus made obesity pretend innocence while normal weight masquerading the hazard. Lean diabetic patients are recently shown to be enriched for known type 2 diabetes risk alleles compared 
to their obese counterparts [32]. On the other end of the spectrum, as they are already subjected to the physiological impact of obesity and insulin resistance, obese individuals presumably need fewer diabetes risk variants to become diabetic [33].

4.4. Implications for Clinicians. The results of studies demonstrating the obesity-paradox have consistently ignited a question of "should we start advising people to become more obese?". We demonstrated, herein, that what previous studies have referred to as "obesity-paradox" is de facto "BMIparadox." Unfortunately, an implicit assumption made in many epidemiologic analyses is that BMI alone is a sufficient measure of obesity effects in regression analyses. Michels et al. have argued that this is not necessarily true and that whether BMI alone adequately captures the effect of anthropometric variables on health outcomes depends on many factors [34]. Inasmuch as BMI harbors an intermixed positive and negative confounding effect on mortality of waist and hip circumference, it cannot be directly translated to obesity. Our finding indicates that if waist circumference constantly increases, BMI does not confer any excess mortality; also, if hip circumference constantly decreases, BMI does not confer any excess mortality. It is prudent, thus, to advise people to try increasing their BMI by gaining weight via physical activities that increase muscle bulks without increasing (or even decreasing) abdominal adiposity. Clinical trials to examine the clinical usefulness of such interventions will be required in the future.

4.5. Unanswered Question. We have previously extensively studied different indices of obesity with respect to health risks in general population. These indices were either directly or inversely associated with mortality [35-39]. To explain excess mortality with lower BMI values, further study is required to find if the obesity-mortality association is confounded by some unmeasured risk factors that tend to accumulate as obesity decreases.

4.6. What Is Already Known on This Topic? Previous studies showed that adults who were of normal weight at the time when they were first diagnosed to have diabetes had higher mortality than their overweight/obese counterparts. Using arbitrary definition for normal-weight, they were not able to discover U-shaped associations.

\section{Conclusion}

Among newly diagnosed diabetic patients, BMI, in its extremes of both leanness and obesity was associated with increased all-cause mortality, independent from established CVD risk factors. However, the association faded when we controlled our multivariate analyses for the confounding bias originating from hip and waist circumference. Our findings indicate that body mass as measured by BMI does not contribute to all-cause mortality independent from central obesity. Rather, BMI harbors intermixed positive and negative confounding effects on waist and hip circumference-related mortality. In particular, failing to control for the confounding effect of hip circumference may stymie unbiased hazard estimation. What previous studies have referred to as the "obesity-paradox" could be reconciled by the fact that BMI is not a perfect measure of obesity. Although not necessarily in the same direction, both body mass and fatness contribute to mortality. Such confounding bias originating from each part should be accounted for while studying obesity-related health risks, since these parameters exhibit covariation in many settings.

\section{Conflict of Interests}

The authors declare that there is no conflict of interests regarding the publication of this paper.

\section{Acknowledgments}

The authors express their appreciation to the participants of district 13 of Tehran for their enthusiastic support in this study. The authors also express their gratitude to Dr. Henry S Kahn (Medical Epidemiologist, Division of Diabetes Translation, Centers for Disease Control \& Prevention, USA) for his thoughtful comments. Mohammadreza Bozorgmanesh designed the study, performed the statistical analysis, interpreted the analyses, and drafted the paper. Farzad Hadaegh interpreted the analyses and revised the paper critically for important intellectual content. Banafsheh Arshi and Farhad Sheikholeslami contributed in the interpretation of the analyses and drafting the paper. Fereidoun Azizi revised the paper critically for important intellectual content. Fereidoun Azizi is the guarantor and takes full responsibility for the work as a whole, including the study design, access to data, and the decision to submit and publish the paper. All authors read and approved the final paper. This study was supported by Grant no. 121 from the National Research Council of the Islamic Republic of Iran.

\section{References}

[1] M. A. Weber, K. Jamerson, G. L. Bakris et al., "Effects of body size and hypertension treatments on cardiovascular event rates: subanalysis of the ACCOMPLISH randomised controlled trial," The Lancet, vol. 381, no. 9866, pp. 537-545, 2013.

[2] D. Schmidt and A. Salahudeen, "The obesity-survival paradox in hemodialysis patients: why do overweight hemodialysis patients live longer?" Nutrition in Clinical Practice, vol. 22, no. 1, pp. 11-15, 2007.

[3] D. S. Schmidt and A. K. Salahudeen, "Cardiovascular and survival paradoxes in dialysis patients: obesity-survival paradoxstill a controversy?" Seminars in Dialysis, vol. 20, no. 6, pp. 486492, 2007.

[4] J. Dhoot, S. Tariq, A. Erande, A. Amin, P. Patel, and S. Malik, "Effect of morbid obesity on in-hospital mortality and coronary revascularization outcomes after acute myocardial infarction in the united states," The American Journal of Cardiology, vol. 111, no. 8, pp. 1104-1110, 2013.

[5] A. de Schutter, C. J. Lavie, D. A. Patel, S. M. Artham, and R. V. Milani, "Relation of body fat categories by Gallagher classification and by continuous variables to mortality in patients with 
coronary heart disease," The American Journal of Cardiology, vol. 111, no. 5, pp. 657-660, 2013.

[6] G. Whitlock, S. Lewington, P. Sherliker et al., "Body-mass index and cause-specific mortality in 900000 adults: collaborative analyses of 57 prospective studies," The Lancet, vol. 373, no. 9669, pp. 1083-1096, 2009.

[7] N. Ruderman, D. Chisholm, X. Pi-Sunyer, and S. Schneider, "The metabolically obese, normal-weight individual revisited," Diabetes, vol. 47, no. 5, pp. 699-713, 1998.

[8] N. B. Ruderman, S. H. Schneider, and P. Berchtold, "The "metabolically-obese," normal-weight individual.," The American Journal of Clinical Nutrition, vol. 34, no. 8, pp. 1617-1621, 1981.

[9] S. Uretsky, S. Bangalore, and F. H. Messerli, "Obesity and mortality: a poorly understood relationship," The American Journal of Cardiology, vol. 99, no. 6, pp. 876-877, 2007.

[10] E. E. Calle, M. J. Thun, J. M. Petrelli, C. Rodriguez, and C. W. Heath Jr., "Body-mass index and mortality in a prospective cohort of U.S. adults," The New England Journal of Medicine, vol. 341, no. 15, pp. 1097-1105, 1999.

[11] S. Uretsky, F. H. Messerli, S. Bangalore et al., "Obesity paradox in patients with hypertension and coronary artery disease," The American Journal of Medicine, vol. 120, no. 10, pp. 863-870, 2007.

[12] C. J. Lavie, R. V. Milani, H. O. Ventura, and A. Romero-Corral, "Body composition and heart failure prevalence and prognosis: getting to the fat of the matter in the 'obesity paradox,"' Mayo Clinic Proceedings, vol. 85, no. 7, pp. 605-608, 2010.

[13] R. F. Kushner and J. L. Roth, "Assessment of the obese patient," Endocrinology and Metabolism Clinics of North America, vol. 32, no. 4, pp. 915-933, 2003.

[14] L. N. McEwen, C. Kim, A. J. Karter et al., "Risk factors for mortality among patients with diabetes: the Translating Research Into Action for Diabetes (TRIAD) Study," Diabetes Care, vol. 30, no. 7, pp. 1736-1741, 2007.

[15] W. Doehner, E. Erdmann, R. Cairns et al., "Inverse relation of body weight and weight change with mortality and morbidity in patients with type 2 diabetes and cardiovascular co-morbidity: an analysis of the PROactive study population," International Journal of Cardiology, vol. 162, no. 1, pp. 20-26, 2012.

[16] W. A. Ray, "Evaluating medication effects outside of clinical trials: new-user designs," American Journal of Epidemiology, vol. 158, no. 9, pp. 915-920, 2003.

[17] M. R. Carnethon, P. J. D. de Chavez, M. L. Biggs et al., "Association of weight status with mortality in adults with incident diabetes," JAMA, vol. 308, no. 6, pp. 581-590, 2012.

[18] F. Azizi, A. Ghanbarian, A. A. Momenan et al., "Prevention of non-communicable disease in a population in nutrition transition: Tehran Lipid and Glucose Study phase II," Trials, vol. 10, article 5, 2009.

[19] D. S. Freedman, H. S. Kahn, Z. Mei et al., "Relation of body mass index and waist-to-height ratio to cardiovascular disease risk factors in children and adolescents: the Bogalusa Heart Study," The American Journal of Clinical Nutrition, vol. 86, no. 1, pp. 3340, 2007.

[20] F. Hadaegh, H. Harati, A. Ghanbarian, and F. Azizi, "Association of total cholesterol versus other serum lipid parameters with the short-term prediction of cardiovascular outcomes: tehran lipid and glucose study," European Journal of Cardiovascular Prevention and Rehabilitation, vol. 13, no. 4, pp. 571-577, 2006.

[21] R. J. Gibbons, J. Abrams, K. Chatterjee et al., "ACC/AHA 2002 guideline update for the management of patients with chronic stable angina-summary article: a report of the American College of Cardiology/American Heart Association Task Force on Practice Guidelines (Committee on the Management of Patients With Chronic Stable Angina)," Circulation, vol. 107, no. 1, pp. 149-158, 2002.

[22] E. Braunwald, E. M. Antman, J. W. Beasley et al., "ACC/AHA guideline update for the management of patients with unstable angina and non-ST-segment elevation myocardial infarction2002: summary article: a report of the American College of Cardiology/American Heart Association Task Force on Practice Guidelines (Committee on the Management of Patients With Unstable Angina)," Circulation, vol. 106, no. 14, pp. 1893-1900, 2002.

[23] American Diabetes Association, "Diagnosis and classification of diabetes mellitus," Diabetes Care, vol. 27, pp. s5-s10, 2004.

[24] B. Balkau, P. Picard, S. Vol, L. Fezeu, and E. Eschwège, "Consequences of change in waist circumference on cardiometabolic risk factors over 9 years: data from an epidemiological study on the insulin resistance syndrome (DESIR)," Diabetes Care, vol. 30, no. 7, pp. 1901-1903, 2007.

[25] F. B. Ortega, D. Lee, P. T. Katzmarzyk et al., “The intriguing metabolically healthy but obese phenotype: cardiovascular prognosis and role of fitness," European Heart Journal, vol. 34, no. 5, pp. 389-397, 2013.

[26] F. Hadaegh, A. Esmaillzadeh, and F. Azizi, "Metabolic risks in individuals with normal body mass index and normal waist circumference," European Journal of Cardiovascular Prevention and Rehabilitation, vol. 14, no. 2, pp. 200-207, 2007.

[27] I. Janssen, P. T. Katzmarzyk, and R. Ross, "Waist circumference and not body mass index explains obesity-related health risk," The American Journal of Clinical Nutrition, vol. 79, no. 3, pp. 379-384, 2004.

[28] Y. D. Tchoukalova, S. B. Votruba, T. Tchkonia, N. Giorgadze, J. L. Kirkland, and M. D. Jensen, "Regional differences in cellular mechanisms of adipose tissue gain with overfeeding," Proceedings of the National Academy of Sciences of the United States of America, vol. 107, no. 42, pp. 18226-18231, 2010.

[29] A. Romero-Corral, V. K. Somers, J. Sierra-Johnson et al., "Accuracy of body mass index in diagnosing obesity in the adult general population," International Journal of Obesity, vol. 32, no. 6, pp. 959-966, 2008.

[30] A. Romero-Corral, V. M. Montori, V. K. Somers et al., "Association of bodyweight with total mortality and with cardiovascular events in coronary artery disease: a systematic review of cohort studies," The Lancet, vol. 368, no. 9536, pp. 666-678, 2006.

[31] D. L. McGee, "Body mass index and mortality: a meta-analysis based on person-level data from twenty-six observational studies," Annals of Epidemiology, vol. 15, no. 2, pp. 87-97, 2005.

[32] B. F. Voight, L. J. Scott, V. Steinthorsdottir et al., “Twelve type 2 diabetes susceptibility loci identified through large-scale association analysis," Nature Genetics, vol. 42, no. 7, pp. 579-589, 2010.

[33] J. R. B. Perry, B. F. Voight, L. Yengo et al., "Stratifying type 2 diabetes cases by BMI identifies genetic risk variants in LAMA1 and enrichment for risk variants in lean compared to obese cases," PLoS Genetics, vol. 8, no. 5, Article ID e1002741, 2012.

[34] K. B. Michels, S. Greenland, and B. A. Rosner, "Does body mass index adequately capture the relation of body composition and body size to health outcomes?" American Journal of Epidemiology, vol. 147, no. 2, pp. 167-172, 1998. 
[35] M. Bozorgmanesh, F. Hadaegh, and F. Azizi, "Diabetes prediction, lipid accumulation product, and adiposity measures; 6year follow-up: Tehran lipid and glucose study," Lipids in Health and Disease, vol. 9, p. 45, 2010.

[36] M. Bozorgmanesh, F. Hadaegh, and F. Azizi, "Predictive performances of lipid accumulation product vs. adiposity measures for cardiovascular diseases and all-cause mortality, 8.6-year follow-up: Tehran lipid and glucose study," Lipids in Health and Disease, vol. 9, article 100, 2010.

[37] M. Bozorgmanesh, F. Hadaegh, and F. Azizi, "Predictive performance of the visceral adiposity index for a visceral adiposityrelated risk: type 2 Diabetes," Lipids in Health and Disease, vol. 10, article 88, 2011.

[38] M. Bozorgmanesh, F. Hadaegh, D. Khalili, and F. Azizi, "Prognostic significance of the complex "Visceral Adiposity Index" vs. simple anthropometric measures: Tehran lipid and glucose study," Cardiovascular Diabetology, vol. 11, p. 20, 2012.

[39] F. Hadaegh, A. Zabetian, P. Sarbakhsh, D. Khalili, W. P. T. James, and F. Azizi, "Appropriate cutoff values of anthropometric variables to predict cardiovascular outcomes: 7.6 years followup in an Iranian population," International Journal of Obesity, vol. 33 , no. 12, pp. 1437-1445, 2009. 


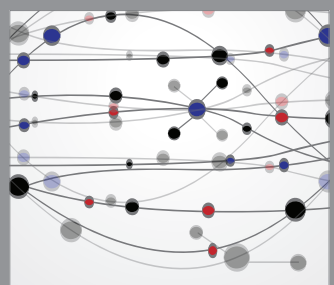

The Scientific World Journal
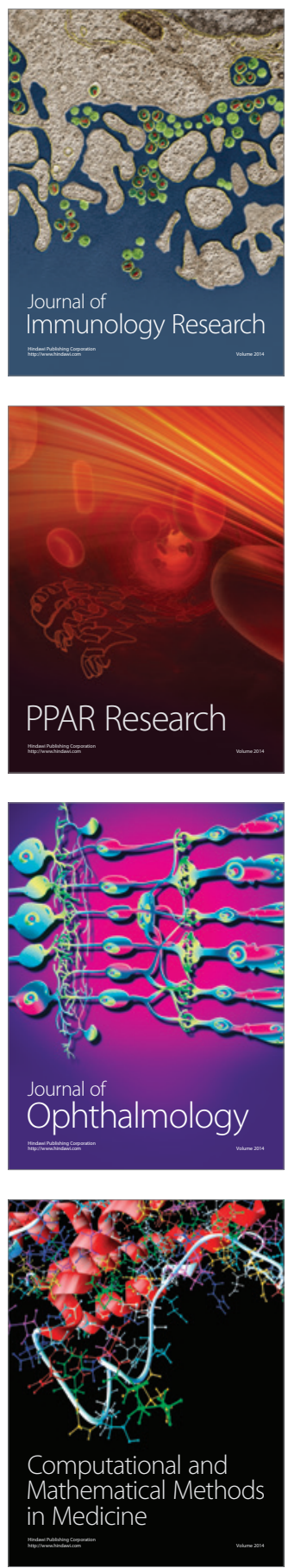

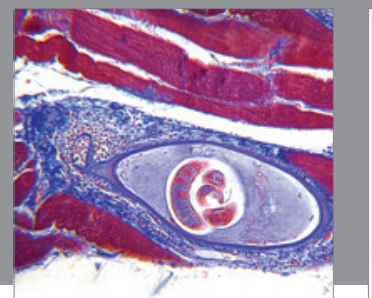

Gastroenterology

Research and Practice
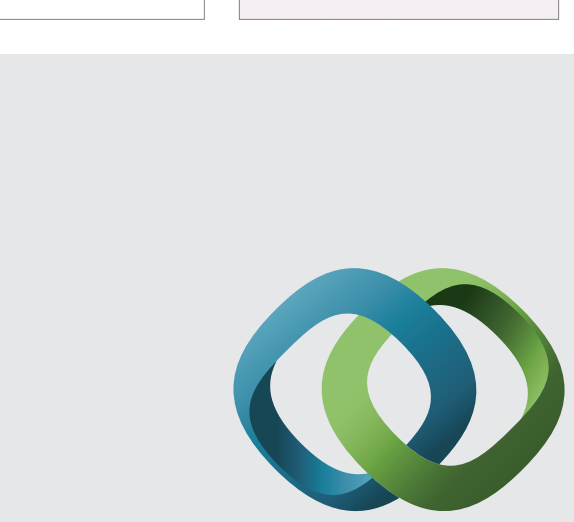

\section{Hindawi}

Submit your manuscripts at

http://www.hindawi.com
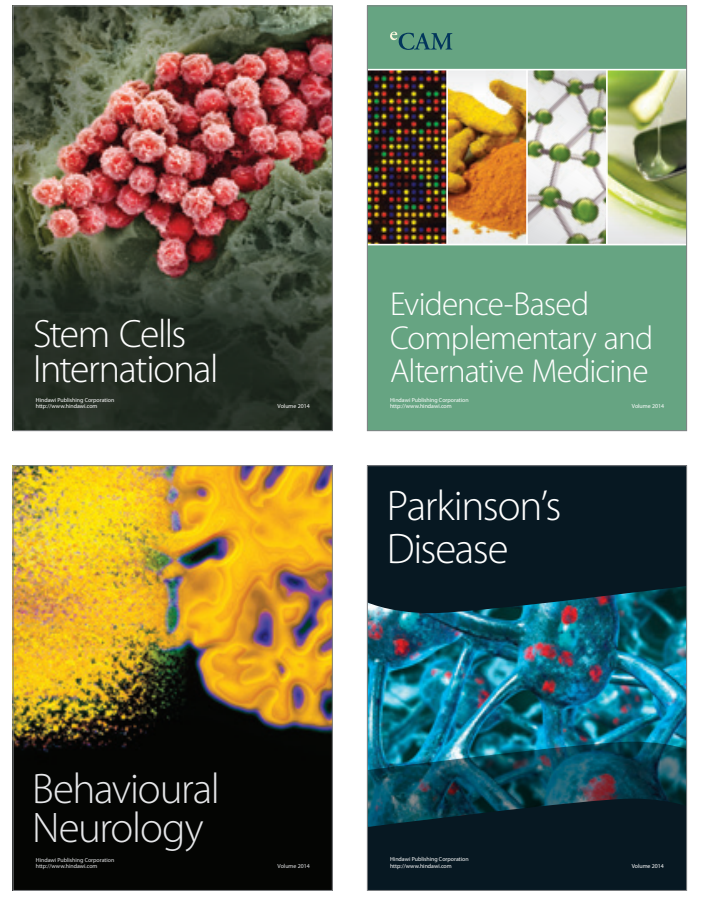
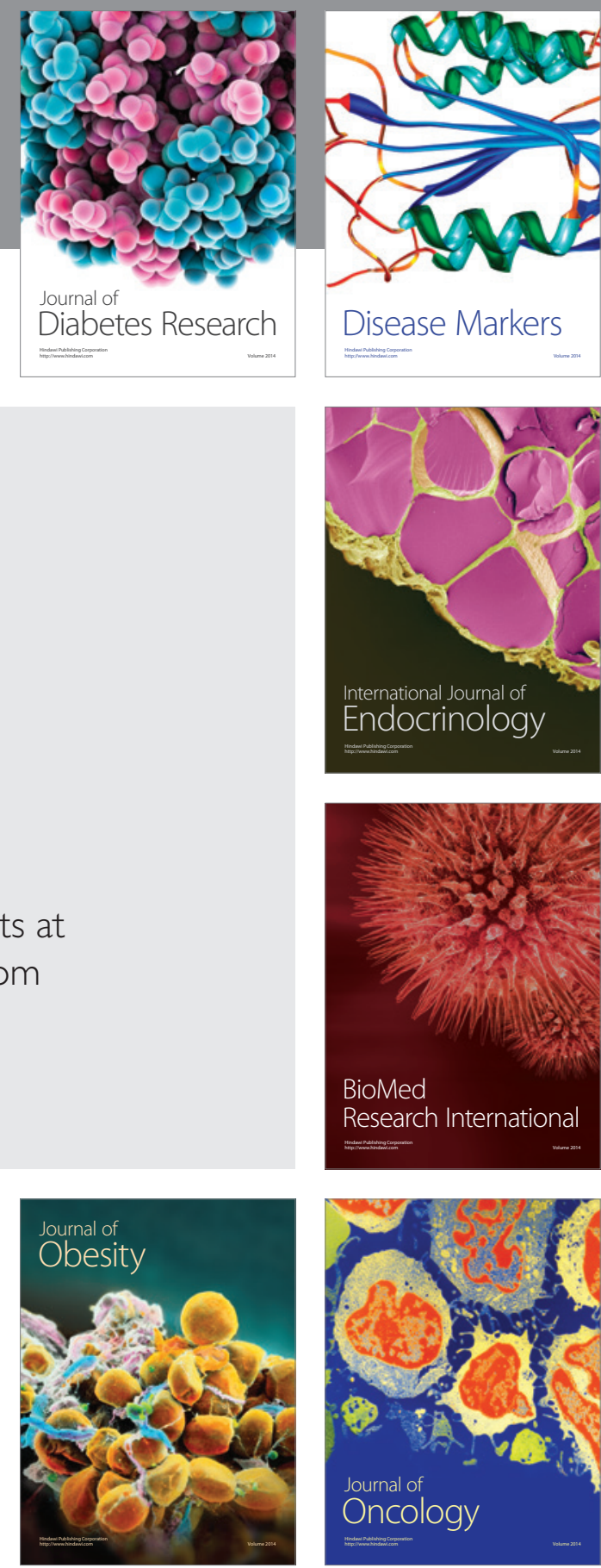

Disease Markers
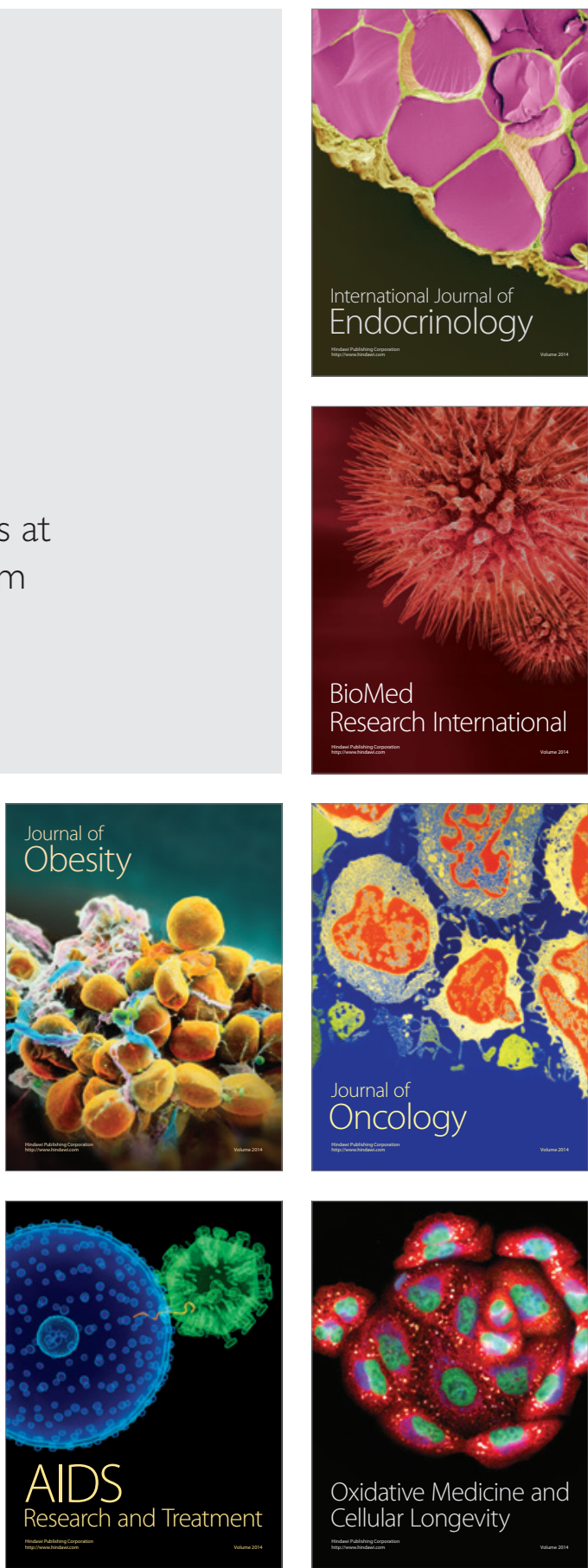